\title{
BMJ Open Management of haemorrhoids: protocol of an umbrella review of systematic reviews and meta-analyses
}

\author{
Min Chen, ${ }^{1}$ Tai-Chun Tang, ${ }^{1}$ Tao-Hong He, ${ }^{1}$ Yong-Jun Du, ${ }^{1}$ Di Qin, ${ }^{2}$ Hui Zheng (1) ${ }^{2}$
}

To cite: Chen M, Tang T-C, He T-H, et al. Management of haemorrhoids: protocol of an umbrella review of systematic reviews and meta-analyses. BMJ Open 2020;10:e035287. doi:10.1136/ bmjopen-2019-035287

- Prepublication history and additional material for this paper are available online. To view these files, please visit the journal online (http://dx.doi. org/10.1136/bmjopen-2019035287).

Received 28 0ctober 2019 Revised 05 March 2020 Accepted 05 March 2020
Check for updates

(C) Author(s) (or their employer(s)) 2020. Re-use permitted under CC BY-NC. No commercial re-use. See rights and permissions. Published by BMJ.

${ }^{1}$ Colorectal disease department, Hospital of Chengdu University of Traditional Chinese Medicine, Chengdu, China

${ }^{2}$ The Third Hospital/Acupuncture and Tuina School, Chengdu University of Traditional Chinese Medicine, Chengdu, China

Correspondence to

Professor Hui Zheng;

zhenghui@cdutcm.edu.cn

\section{ABSTRACT}

Introduction The prevalence of haemorrhoidal diseases was high in general population, and many treatments are proposed for the management of haemorrhoids. The treatments include conservative and surgical interventions; the credibility and strength of current evidence of their effectiveness are not comprehensively evaluated. We aim to evaluate the credibility of systematic reviews and metaanalyses that assess the effectiveness of the treatments for haemorrhoidal diseases through an umbrella review. Methods and analysis We will search Ovid Medline, Embase, Cochrane library and Web of Science from inception to March 2020 without any language restriction. We will include meta-analyses that examine the effectiveness of treatments in the management of haemorrhoids. Two reviewers will independently screen the titles and abstracts of retrieved articles, and they will extract data from the included meta-analyses. For each meta-analysis, we will estimate the effect size of a treatment through the random-effect model and the fixed-effect model, and we will evaluate betweenstudy heterogeneity (Cochrane's $Q$ and $I^{2}$ statistics) and small-study effect (Egger's test); we will also estimate the evidence of excess significance bias. Evidence of each treatment will be graded according to prespecified criteria. Methodological quality of each meta-analysis will be evaluated by using Assessmentof Multiple Systematic Reviews 2. The corrected cover area method will be used to assess the impact of overlap in reviews on the findings of the umbrella review.

Ethics and dissemination We will present the results of the umbrella review at conferences and publish the final report in a peer-reviewed journal. The umbrella review does not require ethical approval.

PROSPERO registration number CRD42019140702.

\section{INTRODUCTION}

Haemorrhoidal disease is one of the most common anorectal conditions encountered in daily practice by general practitioners. ${ }^{1}$ Symptoms related to haemorrhoids include bleeding during or after defecation, pain or discomfort, and perianal itch or irritation. According to a national health survey, an estimated 23 million adults were diagnosed with haemorrhoids in the USA, accounting for $13 \%$ of the US population. Another study from Australian showed that $38.93 \%$ of its

\section{Strengths and limitations of this study}

This will be the first umbrella review that summarises current evidence of using conservative and surgical treatments to treat haemorrhoids.

- The umbrella review will evaluate the overall credibility and strength of the published systematic reviews and meta-analyses that assess treatments for haemorrhoids and classify them into convincing, highly suggestive, suggestive and weak evidence.

- Plentiful treatment options are available for the management of haemorrhoidal diseases, so we might not include all treatments in the umbrella review.

general population suffered from haemorrhoidal diseases. ${ }^{1}$ There were 306000 hospital dischargers and at least 2.2 million outpatient evaluations for haemorrhoids in the $\mathrm{USA},{ }^{23}$ and the demand for haemorrhoidal therapy is predicted to increase. ${ }^{2}$ Although the total annual costs for medications being used to treat haemorrhoid are unclear, one of the popular medications for haemorrhoid, Preparation $H$, was sold for a total cost of US\$136 million in 2017 . $^{4}$

Many treatment options are proposed for the management of haemorrhoids. For lower grade of haemorrhoids (grades I-II by a classification system proposed by Sir Goligher ${ }^{5}$ ), conservative treatments like dietary interventions, lifestyle modification and medication treatment are usually adopted. ${ }^{5}{ }^{6} \mathrm{~A}$ metaanalysis showed that fibre supplementation could reduce the risk of bleeding after defecation. ${ }^{7}$ Another meta-analysis showed that fibre supplementation provided consistent beneficial effect on perianal pain and itching. ${ }^{8}$ Constipation is a known risk factor for the development of haemorrhoids, ${ }^{9}$ and prebiotics and probiotics improve the symptoms of constipation ${ }^{10}$ and therefore are helpful as supplements for managing haemorrhoids. ${ }^{11}$ For medication treatment, topical glucocorticoids, vasoconstrictors like phenylephrinebased suppositories, or analgesics may be 
beneficial for temporarily relieving haemorrhoidal symptoms. ${ }^{6}$ Herbal remedies are also prepared as suppositories or topical agents for alleviating haemorrhoidal symptoms. ${ }^{12}$ Although plentiful options of conservative treatments are available in clinical practice, many of them are not evidence based and the long-term effectiveness of them have not been verified in randomised controlled trials. $^{6}$

For higher grade of haemorrhoids (grades III-IV), surgical interventions are normally suggested; these interventions include rubber band ligation (RBL), stapled haemorrhoidopexy, haemorrhoidectomy and haemorrhoidal artery ligation (HAL). The most commonly used surgical interventions are traditionally excisional haemorrhoidectomy including open (Milligan-Morgan procedure) and closed (Ferguson procedure) haemorrhoidectomy. The most important disadvantage of haemorrhoidectomy is believed to be postoperative complications like postoperative pain and urinary retention. For the consideration of this disadvantage, new surgical procedures like HAL and stapled haemorrhoidopexy are developed to lower the incidence of postoperative complications. However, these new procedures are criticised for high recurrence rate after surgery and high healthcare expenditure, although they reduce the rate of the postoperative complications.

Several meta-analyses comparing the effectiveness of different surgical interventions have been published. ${ }^{13-19}$ The quality of these meta-analyses varied across studies. No systematic review of these meta-analyses has been performed to provide an evidence map for the management of haemorrhoids. Umbrella review is a new systematic review method that quantitatively summarises up-to-date evidence of a specific clinical scenario. ${ }^{20} \mathrm{It}$ provides overview of current evidence and finds out the uncertainty to guide future research. ${ }^{21}$ For the large amount of evidence on conservative and surgical treatments for the management of haemorrhoidal diseases, we aim to conduct an umbrella review to summarise the findings of meta-analyses on conservative and surgical treatments for the management of haemorrhoids and evaluate the strength and credibility of the findings.

\section{METHODS AND ANALYSIS \\ Protocol registration and reporting of findings}

We design the protocol of the review according to the guidelines of Preferred Reporting Items for Systematic Review and Meta-analysis Protocols (PRISMA-P). ${ }^{22}$ The results of the review will be reported according to the recommendation of PRISMA. ${ }^{23}$ Any amendments to the protocol will be recorded and reported in an article with final results. Figure 1 shows the study process.

\section{Patient and public involvement statement}

The study is an umbrella review focusing on the management of haemorrhoids. Patients from the inpatient setting of the colorectal department at Hospital of Chengdu University of Traditional Chinese Medicine participated in the design of outcome assessments.

\section{Inclusion and exclusion criteria}

We will include meta-analyses that examine the effectiveness of lifestyle modification, conservative pharmacological treatments and surgical interventions (vs placebo, sham procedures, usual care or active control) in the management of haemorrhoids. Conservative pharmacological treatments will include oral supplements (insoluble fibres, hydrophilic bulk-forming colloids, prebiotics, probiotics and synbiotics), topical agents (glucocorticoids, vasoconstrictors and analgesics) and phlebotonic drugs comprising flavonoids. We will assess the efficacy and safety of lifestyle modification and conservative pharmacological treatments in the management of grades I-II haemorrhoids. Surgical interventions will include RBL, HAL, sclerotherapy, infrared coagulation, stapled

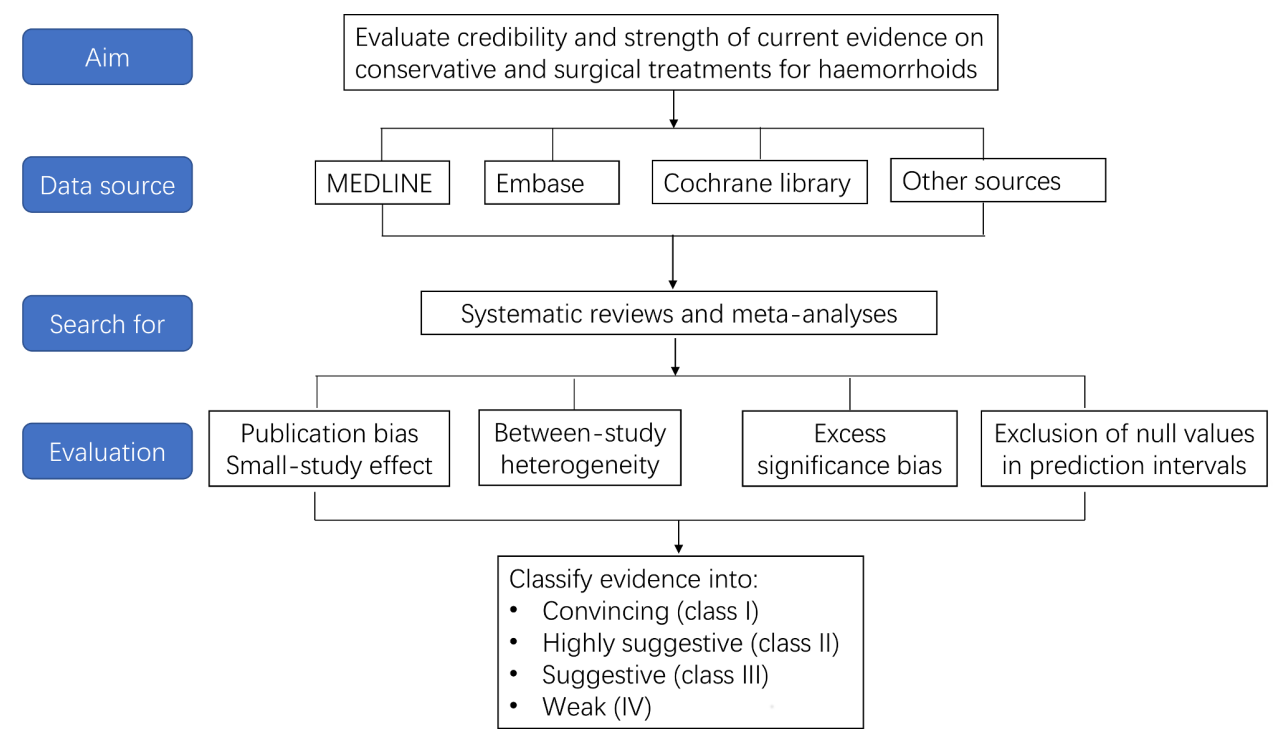

Figure 1 Study flow chart. 
haemorrhoidopexy and haemorrhoidectomy. Metaanalyses of combined treatments (eg, adding a topical bioactive gel to a surgical intervention) will also be evaluated. Outcomes of interest are classified as: symptoms related to haemorrhoids (rectal bleeding, defecation pain and perianal itching with a sense of swelling), surgical related conditions (surgical time, postoperative pain, postoperative bleeding and urinary retention), recurrence of haemorrhoidal symptoms $(<1$ year and $>1$ year $)$ and patient's satisfaction (quality of life, time needed for return to usual activities and hospital stay). We will set no restrictions to the age of participants, study settings (eg, only inpatient or outpatient setting) or the language of publications. When multiple meta-analyses on the same topic (with the same treatment and the same outcomes) were retrieved, we will include the most recent one. We will consider inclusion of ongoing meta-analyses under the condition that primary analyses of these meta-analyses have been finished, and we will contact the authors to ask for related data when possible. We will exclude metaanalyses that report only a summary estimate of effect size (ES) without any further data (eg, mean, SD and the number of events) of the primary studies. We will exclude narrative reviews since they provide no quantitative data (eg, means, SD or event rate) for analysis. We will exclude meta-analyses published in the format of letters to the editor since they usually contain little valuable information.

\section{Outcome assessments}

We will assess the following outcomes: improvement of haemorrhoidal symptoms, surgical related outcomes and patient's satisfaction. The improvement of haemorrhoidal symptoms will be assessed in conservative treatments. The haemorrhoidal symptoms will include rectal bleeding, defecation pain and perianal itching with a sense of swelling. We will adopt the criteria for justification of improvement of haemorrhoidal symptoms as reported in each included systematic review. The surgical related outcomes will be assessed in surgical treatments. These outcomes will include surgical time, postoperative pain, postoperative bleeding, urinary retention and recurrence of haemorrhoidal symptoms $(<1$ year and $>1$ year). The surgical time refers to the duration needed for surgical procedure in managing haemorrhoids, which will be assessed as defined in each systematic review. Postoperative pain will be defined as acute pain at days 1-3 after surgical intervention, including pain during or after defecation, pain during body movement or rest pain at rest. The assessment of the patient's satisfaction includes quality of life, time needed for return to usual activities and hospital stay; it will be assessed in surgical treatments, and it will be assessed at 1 month, 6 months and 12 months after treatment.

\section{Study source and selection process}

We will electronically search Ovid Medline, Embase, the Cochrane Library and Web of Science from inception to

\begin{tabular}{|c|c|}
\hline No. & Search items \\
\hline 1 & hemorrhoid/ \\
\hline 2 & 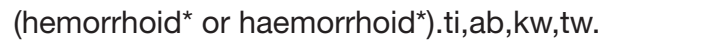 \\
\hline 3 & 1 or 2 \\
\hline 4 & $\begin{array}{l}\text { systematic review/ or meta-analysis/ or systematic } \\
\text { review as topic/ or meta-analysis as topic/ or } \\
\text { network meta-analysis/ }\end{array}$ \\
\hline 5 & (systematic review or meta-analys\$).ti,ab,kw,tw. \\
\hline 6 & 4 or 5 \\
\hline 7 & 3 and 6 \\
\hline
\end{tabular}

March 2020 without any language restriction for potentially eligible candidates. The search strategy will be developed in consultation with an experienced librarian (Yu-Lan Ren from Chengdu University of Traditional Chinese Medicine). In developing the search strategy, four specialists (MC, T-CT, T-HH and Y-JD) from colorectal department in Hospital of Chengdu University of Traditional Chinese Medicine will decide keywords, MeSH (Medical Subject Headings) terms and text words, which will be searched in combination: haemorrhoid, haemorrhoidal, systematic reviews and meta-analyses (table 1 and online supplementary). Additional search will be performed by manual search of the reference lists of the retrieved articles and the search for ongoing meta-analyses registered in PROSPERO or the Cochrane Library. Table 1 shows the strategy developed for searching in Ovid Medline, and the search strategy for Embase and the Cochrane library is shown in the online supplementary. Retrieved articles will be imported into Zotero V.5.0.82 for screening. Two reviewers will independently screen titles and abstracts of retrieved articles according to the inclusion criteria. Fulltext copies will be accessed when the reviewers cannot decide the inclusion of a study through title or abstract screening. The excluded studies will be recorded along with the reason for exclusion at each screening stage. We will contact the corresponding authors of the published articles for additional information if necessary. When there is a disagreement between reviewers on the inclusion of a study, we will solve the problem through group discussion.

\section{Data collection and verification}

Standardised abstraction forms will be used for data collection. Two reviewers (T-CT and MC) will use the forms to collect the following information: characteristics of included meta-analyses (name of first author, publication year, name of intervention, the number of studies included in each meta-analysis, total sample size and the number of meta-analyses), disease conditions (the grading of haemorrhoid ${ }^{24}$ ), intervention and control (name of intervention or control, sample size of each treatment cohort and details of treatment) and outcomes (name and definition of outcome, summary ES and its related 
95\% CI and the number of participants included in the outcome assessment). When the data are only provided through plots, we will use Ycasd ${ }^{25}$ to determine the ES and its $95 \% \mathrm{CI}$; when necessary data were not provided in the article, we will contact the corresponding authors to ask for data. All data will be entered into Epi Info V.7.2 for data analysis. A third reviewer (Y-JD) will check the completeness and correctness of the extracted data.

\section{Assessment of methodological quality}

Methodological quality of the included meta-analyses will be assessed by using the Assessment of Multiple Systematic Reviews 2 (AMSTAR2, an updated version of AMSTAR) tool. ${ }^{26}$ AMSTAR2 has 16 domains; 7 were critical domains, on which the quality rating of an individual systematic review depends. Two reviewers (T-HH and DQ) will rate the quality of each meta-analysis as high, moderate, low and critically low based on the overall score of the AMSTAR2.

\section{Data analysis}

We will use standardised methods adopted by previous umbrella reviews, ${ }^{27-29}$ and state-of-the-art approaches will be used to set criteria to evaluate the credibility of the findings. ${ }^{21}{ }^{29}$ We will first estimate the summary ES and its related 95\% CI using both random-effect and fixedeffect models. Second, we will estimate the $95 \%$ prediction interval (95\% PI) for the summary estimate based on the random-effect model. The $95 \%$ PI specifies the uncertainty for the effect that will be expected in a future study examining the same clinical question. Third, we will evaluate heterogeneity of each meta-analysis by using Cochrane's $Q$ test (considered significant heterogeneity when $\mathrm{p}<0.1)$, and we will classify the degree of heterogeneity into low $\left(\mathrm{I}^{2}<25 \%\right)$, moderate $\left(25 \% \leq \mathrm{I}^{2}<50 \%\right)$, large $\left(50 \% \leq \mathrm{I}^{2}<75 \%\right)$ or very large $\left(\mathrm{I}^{2} \geq 75 \%\right)$ through $\mathrm{I}^{2}$ statistics.

Fourth, we will use Egger's test to evaluate publication bias and small-study effect, and a $p$ value $<0.1$ in the test confirms the bias and small-study effect. Fifth, we will perform an analysis that examines whether the observed number of original studies with positive findings in each meta-analysis is larger than their expected number to detect excess significance bias. ${ }^{30}$ The expected number is calculated as the sum of the statistical power estimates for each original study in a meta-analysis. The power of each study will be calculated through an algorithm using noncentral t-distribution ${ }^{31}{ }^{32}$; the power calculation depends on the value of true ES. Since the true ES for any metaanalysis is impossible to acquire, we will use the ES from the largest study in a meta-analysis to substitute. ${ }^{27}$ We will calculate the ratio of observed number over expected number to evaluate the extent of excess significance bias, and we will claim existence of the bias when a $\chi^{2}$ test arrives at a level of $\mathrm{p}<0.1 .^{33}$

Many systematic reviews and meta-analyses focusing on a similar topic include a different number of primary studies; the overall results and conclusions of an umbrella review might therefore be biased. To assess the potential impact of the overlap in the inclusion of the same primary studies, the degree of overlap within and between reviews was measured using the validated corrected cover area (CCA) method. ${ }^{34}$ A CCA score of $0-5$ indicates slight overlap, $6-10$ moderate, $11-15$ high and $>15$ very high. ${ }^{34}$

\section{Criteria for evaluating credibility of evidence}

We will use the following criteria to evaluate the credibility of the included meta-analyses ${ }^{27} 2835$ : (1) having $\mathrm{p}<10^{-6}$ on the basis of the random-effect model; (2) having $>1000$ participants in a single meta-analysis; (3) having low or moderate heterogeneity $\left(\mathrm{I}^{2}<50 \%\right)$; (4) having 95\% PI that excludes the null value; (5) having no evidence of small-study effect; (6) having no evidence of excess significance bias. Meta-analysis that meets criteria (1)-(6) will be classified as convincing evidence (not suggestive of bias; class I evidence); meta-analysis that meets criteria (1)-(4) will be classified as highly suggestive evidence (class II evidence); meta-analysis that meets criteria (2) and has $\mathrm{p}<0.001$ will be classified as suggestive evidence (class III evidence); meta-analysis that has only $\mathrm{p}<0.05$ will be classified as weak evidence (class IV evidence).

\section{DISCUSSION}

Regarding the high prevalence of haemorrhoids in the general population and its heavy socioeconomic impact, we believe that it is important to provide an evidence map of treatments for haemorrhoids for clinical practitioners and patients, especially when plentiful systematic reviews and meta-analyses are available.

Haemorrhoidal disease is one of the most common clinical conditions in practice. Multiple treatments including conservative and surgical treatments are available for the management of different grades of haemorrhoids. Conservative treatments are usually prescribed for grades I-II haemorrhoids, and surgical treatments are for grades II-IV haemorrhoids. ${ }^{36-39}$ Numerous clinical studies have been performed to study the effect of conservative and surgical treatments on haemorrhoids, ${ }^{56}$ and many systematic reviews and meta-analyses were conducted to evaluate and confirm the effectiveness of these treatments. However, an overview of the systematic reviews and metaanalyses is still lacking. An evidence map of treatments for the management of haemorrhoids is necessary, to facilitate the decision-making of clinical practitioners and participants. Current guidelines usually focus on the evidence of surgical treatments but neglect the evidence of conservative treatments, ${ }^{40}$ which also warrants an overview of current evidence.

Umbrella review is a review of systematic reviews and meta-analyses, which is viewed as one of the four nextgeneration meta-analyses. ${ }^{20}$ An umbrella review is able to quantitatively synthesise information from all systematic reviews and meta-analyses on a given topic. Being different from narrative reviews of systematic reviews, the umbrella review recalculates the ES of a treatment, evaluates the 
credibility of the evidence by estimating excess significance bias and small-study effect and further classifies the confidence of the evidence into four levels. ${ }^{29}{ }^{41}$ Our review will adopt the method of umbrella review, to re-evaluate the credibility of the evidence of treatments for haemorrhoidal diseases, especially the conservative treatments. To the best of our knowledge, the review will be the first to adopt the method of umbrella review in assessing the credibility of current evidence of haemorrhoidal management. The umbrella review is initially designed for confirmation of risk factors in the development of a specific disease condition, ${ }^{20}$ and it is used for evaluating the effectiveness of multiple treatments for a disease in the recent 5 years. ${ }^{27-29}$ We therefore assume that it is essential to use this method to screen and find out treatments that are with convincing evidence for haemorrhoidal management.

The result of this review will be published in a peerreviewed journal, and we believe that the result will benefit clinical practitioners, patients and policy-makers.

\section{Ethics and dissemination}

The study is an umbrella review, which requires no ethical approval. We will presentthe results of the umbrella review at conferences and publish the final report in a peer-reviewed journal.

Acknowledgements The authors thank Yu-Lan Ren, Ling Yue and An-Mei Zhang from Chengdu University of Traditional Chinese Medicine for their suggestions in the search strategy development and data extraction protocol.

Contributors $\mathrm{MC}$ and $\mathrm{HZ}$ designed the study and will perform data analysis. DQ developed the search strategy. MC and T-CT will perform literature search and screen the eligibility of the retrieved articles. T-HH and DQ will evaluate the quality of the retrieved meta-analyses. Y-JD and T-HH will extract information from the eligible studies and prepare the information for data analysis. MC wrote the first draft of the protocol, and all authors read the article and approve it for publication.

Funding The review is financially supported by the National Natural Science Foundation of China (nos. 81774321 and 81473777).

Disclaimer Funders and sponsors have no role in the design of this protocol. Competing interests None declared.

Patient and public involvement Patients and/or the public were involved in the design, or conduct, or reporting, or dissemination plans of this research. Refer to the Methods section for further details.

Patient consent for publication Not required.

Provenance and peer review Not commissioned; externally peer reviewed.

Open access This is an open access article distributed in accordance with the Creative Commons Attribution Non Commercial (CC BY-NC 4.0) license, which permits others to distribute, remix, adapt, build upon this work non-commercially, and license their derivative works on different terms, provided the original work is properly cited, appropriate credit is given, any changes made indicated, and the use is non-commercial. See: http://creativecommons.org/licenses/by-nc/4.0/.

\section{ORCID iD}

Hui Zheng http://orcid.org/0000-0002-0494-1217

\section{REFERENCES}

1 Riss S, Weiser FA, Schwameis K, et al. The prevalence of hemorrhoids in adults. Int $J$ Colorectal Dis 2012;27:215-20.

2 Etzioni DA, Beart RW, Madoff RD, et al. Impact of the aging population on the demand for colorectal procedures. Dis Colon Rectum 2009;52:583-90. discussion 590-591.
3 Davis BR, Lee-Kong SA, Migaly J, et al. The American Society of colon and rectal surgeons clinical practice guidelines for the management of hemorrhoids. Dis Colon Rectum 2018;61:284-92.

4 Sandler RS, Peery AF. Rethinking what we know about hemorrhoids. Clin Gastroenterol Hepatol 2019;17:8-15.

5 Altomare DF, Giuratrabocchetta S. Conservative and surgical treatment of haemorrhoids. Nat Rev Gastroenterol Hepatol 2013;10:513-21.

6 Jacobs D. Clinical practice. hemorrhoids. N Engl J Med 2014;371:944-51.

7 Alonso-Coello P, Guyatt G, Heels-Ansdell D, et al. Laxatives for the treatment of hemorrhoids. Cochrane Database Syst Rev 2005:CD004649.

8 Alonso-Coello P, Mills E, Heels-Ansdell D, et al. Fiber for the treatment of hemorrhoids complications: a systematic review and meta-analysis. Am J Gastroenterol 2006;101:181-8.

9 Peery AF, Sandler RS, Galanko JA, et al. Risk factors for hemorrhoids on screening colonoscopy. PLoS One 2015;10:e0139100.

10 Ford AC, Quigley EMM, Lacy BE, et al. Efficacy of prebiotics, probiotics, and synbiotics in irritable bowel syndrome and chronic idiopathic constipation: systematic review and meta-analysis. $A m \mathrm{~J}$ Gastroenterol 2014;109:1547-61. quiz 1546, 1562.

11 Sakai T, Kubota H, Gawad A, et al. Effect of fermented milk containing Lactobacillus casei strain Shirota on constipation-related symptoms and haemorrhoids in women during puerperium. Benef Microbes 2015;6:253-62.

12 Gan T, Liu Y-D, Wang Y, et al. Traditional Chinese medicine herbs for stopping bleeding from haemorrhoids. Cochrane Database Syst Rev 2010:CD006791.

13 Emile SH, Elfeki H, Sakr A, et al. Transanal hemorrhoidal dearterialization (THD) versus stapled hemorrhoidopexy $(\mathrm{SH})$ in treatment of internal hemorrhoids: a systematic review and meta-analysis of randomized clinical trials. Int $\mathrm{J}$ Colorectal Dis 2019;34:1-11.

14 Vinson-Bonnet B, Higuero T, Faucheron JL, et al. Ambulatory haemorrhoidal surgery: systematic literature review and qualitative analysis. Int J Colorectal Dis 2015;30:437-45.

15 Giordano P, Overton J, Madeddu F, et al. Transanal hemorrhoidal dearterialization: a systematic review. Dis Colon Rectum 2009;52:1665-71.

16 Tjandra JJ, Chan MKY. Systematic review on the procedure for prolapse and hemorrhoids (stapled hemorrhoidopexy). Dis Colon Rectum 2007;50:878-92.

17 Lan P, Wu X, Zhou X, et al. The safety and efficacy of stapled hemorrhoidectomy in the treatment of hemorrhoids: a systematic review and meta-analysis of ten randomized control trials. Int $J$ Colorectal Dis 2006;21:172-8.

18 Nisar PJ, Acheson AG, Neal KR, et al. Stapled hemorrhoidopexy compared with conventional hemorrhoidectomy: systematic review of randomized, controlled trials. Dis Colon Rectum 2004; $47: 1837-45$.

19 Simillis C, Thoukididou SN, Slesser AAP, et al. Systematic review and network meta-analysis comparing clinical outcomes and effectiveness of surgical treatments for haemorrhoids. Br J Surg 2015;102:1603-18

20 loannidis J. Next-generation systematic reviews: prospective metaanalysis, individual-level data, networks and umbrella reviews. $\mathrm{Br} \mathrm{J}$ Sports Med 2017;51:1456-8.

21 Aromataris E, Fernandez R, Godfrey CM, et al. Summarizing systematic reviews: methodological development, conduct and reporting of an umbrella review approach. Int J Evid Based Healthc 2015;13:132-40.

22 Shamseer L, Moher D, Clarke M, et al. Preferred reporting items for systematic review and meta-analysis protocols (PRISMA-P) 2015: elaboration and explanation. BMJ 2015;350:g7647.

23 Moher D, Liberati A, Tetzlaff J, et al. Preferred reporting items for systematic reviews and meta-analyses: the PRISMA statement. PLoS Med 2009;6:e1000097.

24 Rivadeneira DE, Steele SR, Ternent C, et al. Practice parameters for the management of hemorrhoids (revised 2010). Dis Colon Rectum 2011;54:1059-64.

25 Gross A, Schirm S, Scholz M. Ycasd - a tool for capturing and scaling data from graphical representations. BMC Bioinformatics 2014;15:219.

26 Shea BJ, Reeves BC, Wells G, et al. AMSTAR 2: a critical appraisal tool for systematic reviews that include randomised or nonrandomised studies of healthcare interventions, or both. BMJ 2017;358:j4008.

27 Dragioti E, Karathanos V, Gerdle B, et al. Does psychotherapy work? An umbrella review of meta-analyses of randomized controlled trials. Acta Psychiatr Scand 2017;136:236-46. 
$28 \mathrm{He}$ Y, Li X, Gasevic D, et al. Statins and multiple Noncardiovascular outcomes: umbrella review of meta-analyses of observational studies and randomized controlled trials. Ann Intern Med 2018;169:543-53.

29 Khan SU, Khan MU, Riaz H, et al. Effects of nutritional supplements and dietary interventions on cardiovascular outcomes: an umbrella review and evidence MAP. Ann Intern Med 2019;171:190-8.

30 loannidis JPA. Clarifications on the application and interpretation of the test for excess significance and its extensions. J Math Psychol 2013;57:184-7.

31 McShane BB, Böckenholt U. Planning sample sizes when effect sizes are uncertain: the power-calibrated effect size approach. Psychol Methods 2016;21:47-60.

$32 \mathrm{Kim} \mathrm{H}-\mathrm{Y}$. Statistical notes for clinical researchers: sample size calculation 1. comparison of two independent sample means. Restor Dent Endod 2016;41:74-8.

33 Jin S, Li Y-F, Qin D, et al. Non-pharmacological treatments for irritable bowel syndrome: study protocol of an umbrella review of systematic review and meta-analyses. BMJ Open 2019;9:e027778.

34 Pieper D, Antoine S-L, Mathes T, et al. Systematic review finds overlapping reviews were not mentioned in every other overview. $J$ Clin Epidemiol 2014;67:368-75.
35 Belbasis L, Bellou V, Evangelou E, et al. Environmental risk factors and multiple sclerosis: an umbrella review of systematic reviews and meta-analyses. Lancet Neurol 2015;14:263-73.

36 Gallo G, Martellucci J, Sturiale A, et al. Consensus statement of the Italian society of colorectal surgery (SICCR): management and treatment of hemorrhoidal disease. Tech Coloproctol 2020;24:145-64.

37 Higuero T, Abramowitz L, Castinel A, et al. Guidelines for the treatment of hemorrhoids (short report). J Visc Surg 2016;153:213-8.

38 Wald A, Bharucha AE, Cosman BC, et al. ACG clinical guideline: management of benign anorectal disorders. Am J Gastroenterol 2014;109:1141-57. (Quiz) 1058.

39 Madoff RD, Fleshman JW, Clinical Practice Committee, American Gastroenterological Association. American gastroenterological association technical review on the diagnosis and treatment of hemorrhoids. Gastroenterology 2004;126:1463-73.

40 Garg P. Conservative treatment of hemorrhoids deserves more attention in guidelines and clinical practice. Dis Colon Rectum 2018;61:e348.

41 Neuenschwander M, Ballon A, Weber KS, et al. Role of diet in type 2 diabetes incidence: umbrella review of meta-analyses of prospective observational studies. BMJ 2019;366:I2368. 